Pacific Journal of Mathematic 


\title{
SEPARATION AXIOMS AND METRIC-LIKE FUNCTIONS
}

\section{Howard Anton and William J. Pervin}

\begin{abstract}
Boltjanskii has constructed classes of semifield quasipseudo-metrics which are adequate to metrize topological spaces with various separation properties. In this paper we show that his condition given as adequate for $T-0$ spaces actually is satisfied by every semifield metric inducing the topology. On the other hand, we show that the condition he introduced for $T-1$ and $T-2$ spaces is never satisfied by a certain natural semifield quasi-pseudo-metric related to the usual (or Pervin) quasiuniformity. In this paper we completely characterize the classes of semifield quasi-pseudometrics which are not only adequate to metrize $T-1$ and $T-2$ spaces but actually contain all such metrics inducing such topologies. Characterizations of $R-0$ and $R-1$ inducing metrics will also be obtained. Applications to quasi-uniform and quasi-gauge spaces will be made.
\end{abstract}

1. Background. Topological semifields were first introduced by Antonovskii, Boltjanskii, and Sarymsakov in [1, 2, 3]. The reader is referred to these works and to [4] for details; however, for completeness, we will summarize here some of the essential results. Let $\Delta$ be an arbitrary nonempty set. The ring $R^{4}$ of all realvalued functions on $\Delta$ is called a (Tikhonov) semifield if it is given the Tikhonov topology and coordinatewise partial ordering. A semifield quasi-pseudo-metric on an arbitrary set $X$ (over the semifield $R^{\Delta}$ ) is a mapping

$$
d: X \times X \longrightarrow R^{4}
$$

which satisfies the axioms:

M1. $d(x, x)=0$ for any $x \in X$;

M2. $d(x, y) \geqq 0$ for any $x, y \in X$;

M3. $d(x, y)+d(y, z) \geqq d(x, z)$ for any $x, y, z \in X$.

If $x \in X$ and $U$ is an arbitrary neighborhood of zero in the semifield $R^{\Delta}$ then the family of all sets of the form

$$
\{y \in X: d(x, y) \in U\}
$$

is a basis for a topology induced on $X$. Since $R^{4}$ is given the Tikhonov topology, a subbasic neighborhood $U$ of zero is of the form $\pi_{q}^{-1}((-\varepsilon, \varepsilon))$ where $\pi_{q}$ is the $q$ th projection for some $q \in \Delta$ and $\varepsilon>0$ so that the family of all sets of the form 


$$
\Omega(x, \varepsilon, q)=\{y \in X: d(x, y)(q)<\varepsilon\}
$$

is a subbasis for the topology on $X$.

For each semifield quasi-pseudo-metric $d: X \times X \rightarrow R^{4}$ there is a semifield quasi-pseudo-metric $d^{+}: X \times X \rightarrow R^{\Delta+}$ where $\Delta^{+}$is the class of all nonempty finite subsets of $\Delta$ and $d^{+}$is defined by

$$
d^{+}(x, y)\left(q^{+}\right)=\max \left\{d(x, y)(q): q \in q^{+}\right\}
$$

where $q^{+} \in \Delta^{+}$. It is easy to see ([3]) that $d^{+}$, called the directed metric associated with $d$, induces the same topology and quasi-uniformity as $d$, and the family of all sets of the form

$$
\Omega\left(x, \varepsilon, q^{+}\right)=\left\{y \in X: d^{+}(x, y)\left(q^{+}\right)<\varepsilon\right\}
$$

with $\varepsilon>0$ and $q^{+} \in \Delta^{+}$is a base for the topology.

Let $X$ be any topological space and set $\Delta=\{G: G$ is open $\}$. We define a semifield quasi-pseudo-metric $d$ by setting $d(x, y)(G)=1$ if $x \in G$ but $y \notin G$, and $d(x, y)(G)=0$ otherwise. We note that $\{(x, y): d(x, y)(G)<\varepsilon\}=S_{G}$ (in the terminology of [10]) when $0<\varepsilon \leqq 1$ and so this induces the Pervin quasiuniformity for the topology. We will call this semifield quasi-pseudo-metric the Pervin or usual metric.

2. A limitation of Boltjanskii's results; an example. The only aspect of the metrization problem addressed by Boltjanskii in [5] is finding "adequate" conditions with respect to various separation properties. By this is meant finding a condition such that every metric satisfying the condition induces a topology with the property and, as a weak converse, at least one such metric exists. He specifically does not treat the problem of finding conditions on a metric so that, in addition, every metric inducing the topology satisfies the condition.

As an example of this type of result, let us consider the sixth condition of Boltjanskii. To allow for a more consistent treatment with other cases, we shall relabel it $B^{*}$.

$$
B^{*} . d_{\wedge}(x, y)=d(x, y) \wedge d(y, x)=0 \text { only for } x=y \text {. }
$$

(Since the partial ordering is done coordinatewise, the infimum indicated is taken coordinatewise; i.e., we use the equality $(a \wedge b)(q)=$ $\min \{a(q), b(q)\}$ for each $q \in \Delta$.)

This condition is used in his results concerning both T-1 and T-2 spaces. In particular, he proves that $B^{*}$ is an "adequate" condition for the $T-1$ separation property. That this condition is 
not useful as a complete characterization of semifield metrics inducing $T-1$ spaces is clear from the following remarks.

Suppose we want a semifield quasi-pseudo-metric to satisfy condition $B^{*}$. This is equivalent to the requirement that if $x \neq y$ then $d(x, y) \wedge d(y, x) \neq 0$. The conclusion is equivalent to the existence of a $q \in \Delta$ such that $\min \{d(x, y)(q), d(y, x)(q)\} \neq 0$ and so $d(x, y)(q) \neq 0$ and $d(y, x)(q) \neq 0$. In terms of the above usual metric, there must exist an open set $G$ such that $x \in G, y \notin G$ and $y \in G$, $x \notin G$ which is an obvious contradiction. Thus the usual metric for any space can never satisfy $B^{*}$.

Since every topological space is semifield quasi-pseudo-metrizable by the usual metric, a new condition is required if we are to obtain the more complete characterization desired.

3. Characterizations of semifield metrics inducing $T-0$ and $T-1$ topologies. We will now consider the fifth condition of Boltjanskii; which, for consistency, we relabel B0.

B0. $d(x, y) \vee d(y, x)=0$ only for $x=y$.

Boltjanskii has shown that condition B0 is "adequate" for the T-0 separation property. However, the following stronger result holds:

THEOREM 0. Let a topology on $X$ be induced by the semifield quasi-pseudo-metric $d$, then $X$ is T-0 iff $d$ satisfies B0.

Proof. Suppose $x \neq y$. The condition that $X$ be $T-0$ is equivalent to the requirement that there exists some open set $G$ such that $G$ contains $x$ but not $y$, or $G$ contains $y$ but not $x$. This is equivalent to the requirement that there exists a subbasic open set with the same property. In terms of the subbasis $\left(^{*}\right)$, this is equivalent to the existence of some $q \in \Delta$ and an $\varepsilon>0$ such that $y \notin \Omega(x, \varepsilon, q)$ or $x \notin \Omega(y, \varepsilon, q)$. By the definition of these subbasic neighborhoods, we have, equivalently, $d(x, y)(q) \geqq \varepsilon$ or $d(y, x)(q) \geqq \varepsilon$; i.e., $\max \{d(x, y)(q), d(y, x)(q)\} \geqq \varepsilon$. This is equivalent to the requirement that $d(x, y) \vee d(y, x) \neq 0$ and so condition B0.

Let us consider the fourth condition of Boltjanskii which he used in his discussion of complete regularity. For consistency, we will relabel it $\mathrm{B} 1$.

B1. $d(x, y)=0$ only for $x=y$. 
Although Boltjanskii does not use this condition in his results concerning $T-1$ spaces, we have the following:

THEOREM 1. Let a topology on $X$ be induced by the semifield quasi-pseudo-metric $d$, then $X$ is $T-1$ iff $d$ satisfies B1.

Proof. Similar to the above.

4. A new Hausdorff characterization. Let $X$ be a topological space whose topology is induced by the semifield quasi-pseudo-metric $d: X \times X \rightarrow R^{4}$. In terms of the directed metric $d^{+}$associated with $d$ let us define a mapping $d^{*}: X \times X \rightarrow R^{4+}$ by setting

$$
d^{*}(x, y)=\inf \left\{d^{+}(x, z)+d^{+}(y, z): z \in X\right\} .
$$

As was the case with the function $d_{\wedge}$ used by Boltjanskii in his condition $B^{*}$, the function $d^{*}$ is not a metric. We can now formulate our new condition:

B2. $d^{*}(x, y)=0$ only for $x=y$.

We may now prove the following:

THEOREM 2. Let a topology on $X$ be induced by the semifield quasi-pseudo-metric $d$, then $X$ is T-2 iff $d$ satisfies B2.

Proof. $\quad(\mathrm{B} 2 \Rightarrow T-2)$ Let $d$ satisfy $\mathrm{B} 2$ and suppose $x \neq y$. By B2, $d^{*}(x, y) \neq 0$ and so there exists some $q \in \Delta^{+}$such that $d^{*}(x, y)(q) \neq 0$. Hence, for that $q$, we must have inf $\left\{d^{+}(x, z)(q)+d^{+}(y, z)(q)\right.$ : $z \in X\} \neq 0$. Thus, for that $q$ and some $\varepsilon>0$, we have $d^{+}(x, z)(q)+$ $d^{+}(y, z)(q) \geqq 2 \varepsilon$ for every $z \in X$. Equivalently, there exists a $q \in \Delta^{+}$ and an $\varepsilon>0$ such that for every $z \in X$ either $d^{+}(x, z)(q) \geqq \varepsilon$ or $d^{+}(y, z)(q) \geqq \varepsilon$. In terms of the base $\left({ }^{* *}\right)$ for the topology, the sets $\Omega(x, \varepsilon, q)$ and $\Omega(y, \varepsilon, q)$ are open and contain $x$ and $y$ respectively. The condition we have found states that every $z$ fails to belong to one or the other of the sets. Thus they are disjoint, and $X$ is $T-2$.

(T-2 $\Rightarrow$ B2) Let $X$ be $T-2$ and again suppose $x \neq y$. There must exist disjoint open sets $\Omega(x, \varepsilon 1, q 1)$ and $\Omega(y, \varepsilon 2, q 2)$ from the basis $\left({ }^{*}\right)$ containing these points. Disjointness implies that for any $z \in X$ either $d^{+}(x, z)(q 1) \geqq \varepsilon 1$ or $d^{+}(y, z)(q 2) \geqq \varepsilon 2$. Choosing $\varepsilon=2 \min \{\varepsilon 1, \varepsilon 2\}$ and $q=q 1 \cup q 2 \in \Delta^{+}$, we easily see that $d^{+}(x, z)(q)+d^{+}(y, z)(q) \geqq \varepsilon$ for every $z \in X$. Thus $d^{*}(x, y)(q)=\inf \left\{d^{+}(x, z)(q)+d^{+}(y, z)(q): z \in X\right\} \neq 0$, and $d^{*}(x, y) \neq 0$ as desired.

We note that the above proof shows that $d^{*}(x, y)=0$ iff the 
points $x$ and $y$ have no disjoint neighborhoods.

5. Characterizations of $R-0$ and $R-1$ inducing metrics. Although Boltjanskii did not consider the properties $R-0$ and $R-1$ introduced by Davis in [6], we can obtain complete characterizations of these separation axioms in terms of any inducing semifield quasi-pseudometric. We recall that a topological space is $R-0$ iff $x$ belonging to an open set implies that $\bar{x}$ is contained in the open set. The $R-1$ axiom requires that two points with distinct closures be contained in disjoint open sets.

In order to express our results consistently with our previous theorems, we shall introduce the following condition.

D0. $d(x, y)=0$ only for $d(y, x)=0$.

We may now state the following characterization.

THEOREM 3. Let a topology on $X$ be induced by the semifield quasi-pseudo-metric $d$, then $X$ is $R$-0 iff $d$ satisfies D0.

Proof. $(R-0 \Rightarrow \mathrm{D} 0)$ Let $X$ be $R-0$ and suppose $x$ and $y$ are such that $d(y, x) \neq 0$. Then there exists some $q \in \Delta$ and some $\varepsilon>0$ such that $d(y, x)(q)=\varepsilon>0$ and we have $x \notin \Omega(y, \varepsilon, q)$. The complement of the sphere $\Omega(y, \varepsilon, q)$ is closed and does not contain $y$. By $R-0$ there must exist an open set $G$ such that $G$ contains that complement but does not contain $y$. But then $x \in G$ and so $x \in \Omega\left(x, \varepsilon^{\prime}, q^{\prime}\right) \subseteq G$ for some $q^{\prime} \in \Delta^{+}$and $\varepsilon^{\prime}>0$. Since $y \notin G, y \notin \Omega\left(x, \varepsilon^{\prime}, q^{\prime}\right)$; i.e., $d^{+}(x, y)\left(q^{\prime}\right) \geqq \varepsilon^{\prime}$ and so $d(x, y) \neq 0$. Thus $d$ satisfies $\mathrm{D} 0$.

$(\mathrm{D} 0 \Rightarrow R-0)$ Let $x$ belong to an open set $G$. Then $x \in \Omega(x, \varepsilon, q) \subseteq G$ for some $q \in \Delta^{+}$and $\varepsilon>0$. If $y \notin \Omega(x, \varepsilon, q), d^{+}(x, y)(q) \geqq \varepsilon>0$. By D0, $d(y, x) \neq 0$ so $d(y, x)\left(q^{\prime}\right) \geqq \varepsilon^{\prime}$ or $x \notin \Omega\left(y, \varepsilon^{\prime}, q^{\prime}\right)$ for some $q^{\prime} \in \Delta$ and $\varepsilon^{\prime}>0$. Thus $y$ is not in the closure of $x$. It follows that the closure of $x$ is contained in $\Omega(x, \varepsilon, q) \subseteq G$ as desired.

We see immediately that such implications as $T-1=T-0+R-0$ follow from this result. For our treatment of the $R$-1 property we shall introduce the following condition.

D1. $d^{*}(x, y)=0$ only for $d(x, y)=0$.

Before proving the next characterization we note that the closure of a point $x$ is exactly $\{z: d(z, x)=0\}$.

THEOREM 4. Let a topology on $X$ be induced by the semifield 
quasi-pseudo-metric $d$, then $X$ is $R$-1 iff $d$ satisfies D1.

Proof. $(R-1 \Rightarrow \mathrm{D} 1)$ Let $d(x, y) \neq 0$ for some $x, y \in X$. As noted above, $x \notin \bar{y}$. Since $x \in \bar{x}$, we have $\bar{x} \neq \bar{y}$. By $R-1, x$ and $y$ have disjoint neighborhoods which, by the remark following Theorem 2, implies that $d^{*}(x, y) \neq 0$.

(D1 $\Rightarrow R-1$ ) Suppose $x$ and $y$ have no disjoint neighborhoods. Again, this implies that $d^{*}(x, y)=0$. By $\mathrm{D} 1$, we have $d(x, y)=0$. From the symmetry of $d^{*}$ it follows that $d(y, x)=0$. By the above remark, both $x \in \bar{y}$ and $y \in \bar{x}$. It follows that $\bar{x}=\bar{y}$ as desired.

We see immediately that such implications as $T-2=T-1+R-1$ and $R$-1 implies $R-0$ follow immediately.

6. Application to quasi-uniform spaces. Since every quasiuniform space is semifield quasi-pseudometrizable (the usual or Pervin semifield quasi-pseudo-metric induces the quasi-uniformity), we can now apply our results from the previous sections to the case of quasi-uniform spaces. For quasi-uniform T-0 spaces, Murdeshwar and Naimpally [9, p. 35] have given two characterizations, one in terms of the quasiuniformity $\mathbb{Q}$ and the other in terms of an inducing (real) quasi-pseudo-metric $d$.

(i ) $(X, \mathbb{Q})$ is $T-0$ iff $\cap\{U: U \in \mathbb{Q}\}$ is anti-symmetric.

(ii) $(X, d)$ is $T-0$ iff $d(x, y)+d(y, x)=0$ only for $x=y$.

It is clear that our Theorem 0 yields the second of these results immediately. In the case of $T-1$ spaces, again there are two characterizations $[9$, p. 36]:

(i ) $(X, \mathscr{Q})$ is $T-1$ iff $\cap\{U: U \in \mathbb{Q}\}=\mathfrak{\Im}_{X}$ (the diagonal).

(ii) $(X, d)$ is $T-1$ iff $d(x, y)=0$ only for $x=y$.

Again it is clear that our Theorem 1 yields the second result in this case. For T-2 spaces, however, Murdeshwar and Naimpally [9, p. 36] give only the single condition:

(i ) $(X, \mathbb{Q})$ is $T-2$ iff $\cap\left\{U^{-1} \circ U: U \in \mathbb{Q}\right\}=\mathfrak{\Im}_{X}$.

Using our Theorem 2 we can now give a condition for $T-2$ in terms of an inducing (real) quasi-pseudo-metric $d$. For a real quasi-pseudometric, $d^{+}=d$ and so condition $\mathrm{B} 2$ reduces to

$$
\inf \{d(x, z)+d(y, z): z \in X\}=0 \text { only for } x=y \text {, }
$$

and we obtain the result

(ii) $(X, d)$ is $T-2$ iff $\inf \{d(x, z)+d(y, z): z \in X\}=0$ only for $x=y$, or, equivalently.

(iii) $(X, d)$ is $T-2$ iff $d^{*}(x, y)=0$ only for $x=y$. 
In the case of $R-0$ spaces, again there are two characterizations [9, p. 38]:

(i) $(X, \mathbb{Q})$ is $R-0$ iff $\cap\{U: U \in \mathbb{Q}\}$ is symmetric.

(ii) $(X, d)$ is $R-0$ iff $d(x, y)=0$ iff $d(y, x)=0$.

It is clear that our Theorem 3 yields the second result in this case. For $R-1$ spaces, however, Murdeshwar and Naimpally [9, p. 40] give only the single condition:

(i ) $\left(X, \mathbb{Q}^{\prime}\right)$ is $R-1$ iff $\cap\left\{U^{-1} \circ U: U \in \mathbb{Q}\right\}=\{(x, y): \bar{x}=\bar{y}\}$. Using our Theorem 4 we can now give a condition for $R$-1 in terms of an inducing (real) quasi-pseudo-metric $d$ as follows:

(ii) $(X, d)$ is $R$-1 iff $d^{*}(x, y)=0$ only for $d(x, y)=0$. This extends the earlier results also given in [8].

7. Application to quasi-gauge spaces. In his thesis [11] (see also [12,13]), Reilly has generalized many results concerning gauge spaces (see [7]). Reilly defines a quasi-gauge space to be a set with a family $\mathscr{P}$ of (real) quasi-pseudo-metrics inducing a topology by taking as a subbase the family of all spheres $S(x, \varepsilon, p)=\{y: p(x, y)<\varepsilon\}$, where $p \in \mathscr{P}$ and $\varepsilon>0$. Clearly, the notion of a semifield-valued quasi-pseudo-metric is equivalent to this and may allow a more convenient notation and terminology.

Reilly calls a quasi-gauge $\mathscr{P}$ on a set $X$ augmented if for any two elements $p^{1}$ and $p^{2}$ of $\mathscr{P}$ there exists an element $p^{3}$ in $\mathscr{P}$ such that

$$
p^{3}(x, y) \geqq \max \left\{p^{1}(x, y), p^{2}(x, y)\right\}
$$

for all $x, y \in X$. Further, the augmented quasi-gauge $\mathscr{P}^{+}$determined by $\mathscr{P}$ is the family of all quasi-pseudo-metrics of the form $\max \left\{p^{1}, \cdots, p^{n}\right\}$, where $\left\{p^{1}, \cdots, p^{n}\right\}$ is a finite subset of $\mathscr{P} . \mathrm{He}$ shows that the family of all spheres using $\mathscr{P}^{+}$determines a base rather than just a subbase for the topology for $X$.

It is clear that the augmented quasi-gauges are just the directed semifield metrics we have used above. Other results given by Reilly are easy to state in terms of semifield metrics. The fact that every topological space is a quasi-gauge space is equivalent to the result that every topological space is semifield metrizable; indeed, the usual metric introduced above is based on Reilly's usual quasi-gauge.

From the identification between quasi-gauges and semifield metrics, it follows that the separation axioms $T-0, T-1, T-2, R-0$, and $R-1$ could be characterized in quasi-gauge spaces by the corres- 
ponding translated versions of properties B0, B1, B2, D0, and D1. We will not carry out the details of this translation.

\section{REFERENCES}

1. M. Ja. Antonovskii, V. G. Boltjanskii, T.A. Sarymsakov, Topological Semifields, Tashkent, 1960.

2. - Metric Spaces Over Semifields, Tashkent, 1961.

3. - Topological Boolean Algebras, Tashkent, 1963.

4. - An outline of the theory of topological semifields, Russian Math. Surveys, 21 (1966), 163-192.

5. V.G. Boltjanskii, Separability Axioms and a Metric, Dokl. Akad. Nauk SSSR, 197 (1971) = Soviet Math. Dokl., 12 (1971), 639-643.

6. A. S. Davis, Indexed systems of neighborhoods for topological spaces, Amer. Math. Monthly, 68 (1961), 886-893.

7. J. Dugundji, Topology, Allyn \& Bacon, Boston, 1967.

8. M. G. Murdeshwar, S. A. Naimpally, Trennungsaxioms in quasi-uniform spaces, Nieuw Archief voor Wiskunde, (3) 14 (1964), 97-101.

9. M. G. Murdeshwar, S. A. Naimpally, Quasi-uniform Topological Spaces, Noordhoff, Groningen, 1966.

10. W. J. Pervin, Quasi-uniformization of topological spaces, Math. Annalen, 147 (1962), 316-317.

11. I. L. Reilly, Quasi-gauges, quasi-uniformities, and bitopological spaces, Thesis, University of Illinois at Urbana-Champaign, 1970.

12. - On generating quasi uniformities, Math. Ann., 189 (1970), 317-318.

13. - Quasi-gauge spaces, J. London Math. Soc., (2) 6 (1973), 481-487.

Received February 18, 1976.

UNIVERSITY OF TEXAS

AND

DREXEL UNIVERSITY 


\section{PACIFIC JOURNAL OF MATHEMATICS}

\section{EDITORS}

RICHARD ARENS (Managing Editor)

University of California

Los Angeles, California 90024

\section{R. A. Beaumont}

University of Washington

Seattle, Washington 98105
J. DugundjI

Department of Mathematics

University of Southern California

Los Angeles, California 90007

D. Gilbarg and J. Milgram

Stanford University

Stanford, California 94305

\section{ASSOCIATE EDITORS}
E. F. BECKENBACH
B. H. NeumanN
F. WOLF
K. YosHIDA

\section{SUPPORTING INSTITUTIONS}

\author{
UNIVERSITY OF BRITISH COLUMBIA \\ CALIFORNIA INSTITUTE OF TECHNOLOGY \\ UNIVERSITY OF CALIFORNIA \\ MONTANA STATE UNIVERSITY \\ UNIVERSITY OF NEVADA \\ NEW MEXICO STATE UNIVERSITY \\ OREGON STATE UNIVERSITY \\ UNIVERSITY OF OREGON \\ OSAKA UNIVERSITY
}

\author{
UNIVERSITY OF SOUTHERN CALIFORNIA \\ STANFORD UNIVERSITY \\ UNIVERSITY OF HAWAII \\ UNIVERSITY OF TOKYO \\ UNIVERSITY OF UTAH \\ WASHINGTON STATE UNIVERSITY \\ UNIVERSITY OF WASHINGTON \\ AMERICAN MATHEMATICAL SOCIETY
}

The Supporting Institutions listed above contribute to the cost of publication of this Journal, but they are not owners or publishers and have no responsibility for its content or policies.

Mathematical papers intended for publication in the Pacific Journal of Mathematics should be in typed form or offset-reproduced, (not dittoed), double spaced with large margins. Please do not use built up fractions in the text of your manuscript. You may however, use them in the displayed equations. Underline Greek letters in red, German in green, and script in blue. The first paragraph or two must be capable of being used separately as a synopsis of the entire paper. Items of the bibliography should not be cited there unless absolutely necessary, in which case they must be identified by author and Journal, rather than by item number. Manuscripts, in triplicate, may be sent to any one of the editors. Please classify according to the scheme of Math. Reviews, Index to Vol. 39. All other communications should be addressed to the managing editor, or Elaine Barth, University of California, Los Angeles, California, 90024.

The Pacific Journal of Mathematics expects the author's institution to pay page charges, and reserves the right to delay publication for nonpayment of charges in case of financial emergency.

100 reprints are provided free for each article, only if page charges have been substantially paid. Additional copies may be obtained at cost in multiples of 50 .

The Pacific Journal of Mathematics is issued monthly as of January 1966. Regular subscription rate: $\$ 72.00$ a year (6 Vols., 12 issues). Special rate: $\$ 36.00$ a year to individual members of supporting institutions.

Subscriptions, orders for back numbers, and changes of address should be sent to Pacific Journal of Mathematics, 103 Highland Boulevard, Berkeley, California, 94708.

PUBLISHED BY PACIFIC JOURNAL OF MATHEMATICS, A NON-PROFIT CORPORATION

Printed at Kokusai Bunken Insatsusha (International Academic Printing Co., Ltd.), 8-8, 3-chome, Takadanobaba, Shinjuku-ku, Tokyo 160, Japan. 


\section{Pacific Journal of Mathematics \\ Vol. 65, No. $2 \quad$ October, 1976}

Andrew Adler, Weak homomorphisms and invariants: an example .......... 293

Howard Anton and William J. Pervin, Separation axioms and metric-like

functions ............................................. 299

Ron C. Blei, Sidon partitions and p-Sidon sets .................... 307

T. J. Cheatham and J. R. Smith, Regular and semisimple modules ........... 315

Charles Edward Cleaver, Packing spheres in Orlicz spaces .............. 325

Le Baron O. Ferguson and Michael D. Rusk, Korovkin sets for an operator on a

space of continuous functions ............................. 337

Rudolf Fritsch, An approximation theorem for maps into Kan fibrations ....... 347

David Sexton Gilliam, Geometry and the Radon-Nikodym theorem in strict

Mackey convergence spaces .................................

William Hery, Maximal ideals in algebras of topological algebra valued

functions ...................................... 365

Alan Hopenwasser, The radical of a reflexive operator algebra ........... 375

Bruno Kramm, A characterization of Riemann algebras................. 393

Peter K. F. Kuhfittig, Fixed points of locally contractive and nonexpansive

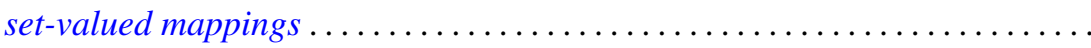

Stephen Allan McGrath, On almost everywhere convergence of Abel means of

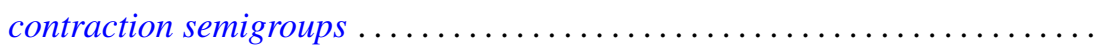

Edward Peter Merkes and Marion Wetzel, A geometric characterization of

indeterminate moment sequences............................ 409

John C. Morgan, II, The absolute Baire property ................... 421

Eli Aaron Passow and John A. Roulier, Negative theorems on generalized convex approximation .................................... 437

Louis Jackson Ratliff, Jr., A theorem on prime divisors of zero and characterizations of unmixed local domains ..............

Ellen Elizabeth Reed, A class of $T_{1}$-compactifications................... 471

Maxwell Alexander Rosenlicht, On Liouville's theory of elementary

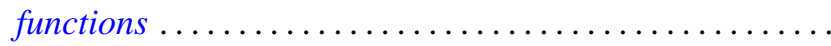

Arthur Argyle Sagle, Power-associative algebras and Riemannian

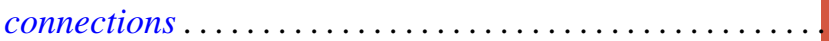

Chester Cornelius Seabury, On extending regular holomorphic maps from Stein manifolds...

Elias Sai Wan Shiu, Commutators and numerical ranges of powers of operators ...................................

Donald Mark Topkis, The structure of sublattices of the product of $n$ lattices ... 525

John Bason Wagoner, Delooping the continuous $K$-theory of a valuation

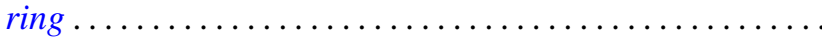

Ronson Joseph Warne, Standard regular semigroups...........

Anthony William Wickstead, The centraliser of $E \otimes_{\lambda} F \ldots$. 